\title{
In memoriam Michel Aufray (1949-2007)
}

Jacqueline de La Fontinelle

\section{Q OpenEdition \\ Journals}

\section{Édition électronique}

URL : http://journals.openedition.org/jso/1118

DOI : $10.4000 /$ jso. 1118

ISSN : 1760-7256

Éditeur

Société des océanistes

\section{Édition imprimée}

Date de publication : 1 décembre 2007

Pagination : 347-351

ISBN : 978-2-85430-010-9

ISSN : 0300-953x

Référence électronique

Jacqueline de La Fontinelle, «In memoriam Michel Aufray (1949-2007) 》, Journal de la Société des

Océanistes [En ligne], 125 | Année 2007-2, mis en ligne le 26 mai 2008, consulté le 15 septembre 2020. URL : http://journals.openedition.org/jso/1118

(C) Tous droits réservés 


\section{In memoriam Michel Aufray (1949-2007)}

par

Jacqueline de La FONTINELLE*

Michel Aufray est mort le 22 août 2007 dans un accident de voiture survenu dans l'Ain sur une petite route de campagne... Il avait 58 ans.

Lorsqu'Isabelle Leblic, quelques jours après l'annonce de cette nouvelle si difficile à croire, m'a demandé si je voulais faire un article sur Michel pour les Océanistes, j'ai répondu « oui », très reconnaissante, avec l'absurde idée de faire encore quelque chose pour lui...

Maintenant, je mesure la difficulté de la tâche : rendre justice à sa carrière, à ses enseignements, à ce qu'il a apporté à la connaissance de la littérature orale du Pacifique et fait pour la reconnaissance des langues et cultures locales, à son dévouement aux siens et à sa fidélité envers ses amis... et tenter de témoigner de mon affection, de notre affection puisque pour mes enfants qui le connaissent depuis 1970, Michel était un parent très cher.

Je ne peux qu'évoquer dans les lignes qui suivent quelques souvenirs, tenter de faire vivre des images de son souvenir et réserver à un temps ultérieur l'écriture d'un hommage plus classique.

Qui de nous qui l'avons connu, à l'évocation de son nom, ne peut d'abord voir s'avancer avec une surprenante grâce légère ce personnage rebondi au visage de poupon rieur ? Prêt à décocher une plaisanterie, une remarque inattendue, drolatique? Car Michel avait vraiment l'esprit « estudiantin », le goût du canular et du burlesque. Il aimait à se définir comme un « maître de circonférence » et lui, qui, faisant rire n'était pourtant jamais méchant, se moquait souvent cruellement de sa « rebondité ». Je l'avais connu, étudiant, en 1970, mince jeune homme blond qui jouait les jeunes premiers dans les pièces montées par ses copains. À cette époque, il était inscrit à l'Institut d'Études scandinaves. Avec son ami Philippe Martel, ils avaient racheté le camion de la blanchisseuse du Plessis-Robinson! (Eh oui ! en ce temps-là, les blanchisseurs venaient chercher le linge et le rapportaient propre et repassé !). Se nourrissant de conserves (pour éviter d'acheter les denrées trop chères pour leurs maigres économies), malgré les pannes, ils avaient visité la Norvège.

Mais les blondes sirènes scandinaves ont été oubliées pour les mystérieuses déités austronésiennes.

Michel, en effet, à la suite du pari fait avec ses amis de s'inscrire à un cours "farfelu» d'une langue inconnue, avait choisi, à l'INALCO, le «houailou» (l'ajië, Sud de la NouvelleCalédonie), sans avoir la moindre idée du lieu où était parlée cette langue. C'est ainsi que Michel fut mon premier étudiant alors que je venais de prendre la succession des Leenhardt, père et fils, Maurice et Raymond.

Michel va se passionner pour les langues alors enseignées dans ce qui devint la section de Langues océaniennes, le tahitien (enseigné par la pittoresque $\mathbf{M}^{\mathrm{me}}$ Norman qui n'admettait pas que le secrétariat refuse d'enregistrer les 21 et 22 qu'elle attribuait à ses élèves), le houailou (avec moi !) et le drehu (langue de Lifou dont Wamo Haocas créa le premier enseignement). Il renonce aux langues scandinaves pour se consacrer à l'Océanie. Il va suivre tous les cours et obtenir toutes les unités de valeur de « langues et de civilisation ", puis tous les diplômes, avant de devenir chargé de cours, assistant, maître de conférence, en fait mon "co-équipier », ensei-

*jdelafontinelle@lagoon.nc 
gnant et créateur d'enseignements fondés sur ses acquis et ses recherches. Ayant gravi tous les degrés, soutenu brillamment l'une des dernières thèses d'État (supprimées en 1982), il sera mon successeur en 2001 comme professeur des universités à la chaire de « linguistique et littératures du Pacifique ». Chaire qui disparaît avec lui...

Pour payer ses études, Michel avait été " pion » et avait enseigné le français, à SainteMarie à Antony, chez les curés où il avait été élève ; il fut aussi veilleur de nuit, empaqueta des yaourts, de nuit, chez Danone et, pour assouvir sa passion naissante de bibliophile, dès qu'il fut « secrétaire » chez André-Georges Haudricourt, notre « patron », il se passait de déjeuner !

Haudricourt avait choisi comme terrain de recherches le Pacifique et, directeur de recherches au CNRS, il avait droit à des « techniciens ». Michel, dans l'appartement de la Rue d'Assas, partageait avec $\mathbf{M}^{\text {me }}$ Dufour, dans l'incroyable amoncellement de livres et de revues entassés jusqu'à mi-hauteur des fenêtres, un espace exigu où tous deux classaient, annotaient les travaux des chercheurs sur les langues de NouvelleCalédonie (1973-1982).

C'est grâce à Haudricourt, par le biais d'une mission CNRS, que Michel a eu la possibilité de mener sa première enquête en Océanie. De retour du " terrain » pacifique, il rédige sa thèse de $3^{\mathrm{e}}$ cycle Phonologie et description grammaticale de l'anecom (Archipel du Vanuatu) qu'il soutient en 1981. Il apprit aussi avec ravissement (!) à l'occasion de son séjour vanuatais que les " gros », les gens de « poids » sont enviés et respectés en Océanie traditionnelle... et cela lui donna une remarquable confiance dans ses rapports avec des gens pas toujours bien intentionnés envers « les blancs ».

C'est grâce à Haudricourt aussi, que Michel a été " mis » à la botanique. Rien ne contrariait davantage "le patron » que le non-intérêt au monde concret et par-dessus tout, l'ignorance du nom scientifique et des usages de la Plante. Corollaire de la vision matérialiste des « Rationalistes ", cette approche des autres cultures a été déterminante pour tous les jeunes chercheurs. Haudricourt, bourru et tendre, enseignait d'une façon toute particulière, bousculant ses étudiants, enchantés. Ses cours d'ethnobotanique se faisaient, soit dans les locaux de Cuvier et dans les serres du Jardin des Plantes, soit lors de sorties botaniques... À sa dernière mission d'enseignement à Nouméa, en juin 2007, Michel m'emmenait voir une plante de "brousse", poussée là, «par erreur », le long du mur d'un jardin, et nous discutions avec passion de l'arrivée puis du recul des oxalis roses importés invo- lontairement et fleurissant les fossés de « l'exterritoriale ».

Chez Haudricourt, Michel a rencontré les chercheurs Denise et Lucien Bernot, Georges Condominas, Jean Guiart dont il suivit les séminaires, $\mathrm{M}^{\mathrm{me}}$ Bruhnes-Delamarre qui fit ce merveilleux exposé lors de la remise d'un prix CNRS pour les travaux du « patron ». Comment ne pas ici évoquer l'exquise soupe d'épeautre que le professeur du Collège de France, Lucien Bernot, préparait et nous invitait à partager avec Haudricourt, Bazin (l'éminent turcologue, grand maître de la contrepèterie), Monique son épouse et quelques spécialistes du birman. Michel, fin gourmet et connaisseur de bons vins, participait autant aux discussions culinaires qu'aux comparaisons ethnolinguistiques de nos hôtes. Ces dîners à Antony, l'hospitalité de Denise courant de la cuisine à la table, l'œil au confort de tous, tout en discutant une entrée de son dictionnaire birman avec Haudricourt! Simplicité, rires, humour et savoirs... chaleureux souvenirs!

Michel s'intéressait à tout, lisant " en biais » et à une vitesse incroyable, et il ne cessa jamais d'arrondir joliment son pécule de connaissances. Il adorait la poésie et savait des milliers de vers, des poètes surréalistes aussi bien que des tirades du Cid, des pages de Chateaubriand... Il marchait aussi des kilomètres, une énorme sacoche de livres au côté et découvrait Paris quartier par quartier. En quelque sorte, il « vivait» dans le quartier qu'il s'était choisi. Épiceries, marchés, coiffeurs, libraires et bouquinistes... et, bien sûr, petits restaurants, le voyaient revenir jour après jour, jusqu'à ce qu'il estime avoir suffisamment bonne connaissance du lieu pour partir en explorer un autre! mais lorsqu'il y revenait pour vous faire partager son enthousiasme et, discrètement, ses connaissances historiques, vous découvriez qu'il y avait laissé des amis ravis de le revoir.

Le début de sa carrière si riche en découvertes fut un temps très difficile matériellement pour Michel : le ministère, pourtant sollicité par les DOM-TOM, ne se décidait pas à accorder le renouvellement d'un poste de maître de conférences que Michel occuperait - enfin, à, son retour de mission en Nouvelle-Calédonie, en 1984. Les « Langues $\mathrm{O}$ » avaient un enseignement nouveau à offrir, celui de « littérature orale du Pacifique ».

Les Langues $\mathrm{O}$ !, « la maison » comme nous disions, comme il a été heureux et fier d'en faire partie - sentiment archaïque et dépassé, mais qui « portait » et poussait à donner le meilleur de soi-même... jusque dans les salons de la rue de Lille où se tenaient les interminables et multiples 
conseils dont Michel fut membre, président... C'est aussi dans ces salons que Michel Aufray et Michel Perret, collègues et amis, organisèrent les « déjeuners culturels » (souvent savoureux, parfois fort inattendus) pour accompagner la rédaction de La cuisine d'Orient. Chaque secteur linguistique de L.O. avait été sollicité pour contribuer à cette publication, par un article, évidemment, mais aussi par des réalisations culinaires (avouons que bien des ingrédients ne purent être fournis, tels les morceaux d'hippopotames, d'éléphants ou de phoque vieux de six mois...).

En tentant d'esquisser le parcours de Michel, je me rends compte combien il m'est difficile de ne pas écrire « nous ", car, en fait, après sa nomination, nous partagions créations et enseignements, lourds travaux administratifs, efforts pour développer les cursus, pour établir et assurer les diplômes qui donneraient une assise et un " poids » aux Langues océaniennes. En effet, il nous semblait alors, et cette conviction va être celle de Michel jusqu'au bout, qu'il est impératif de rendre aux peuples océaniens un peu de ce qui leur a été pris, l'idée que la reconnaissance des langues et des valeurs culturelles locales est un facteur déterminant de l'évolution. C'est grâce à la ténacité de notre section que la mention de Langues $O$ «jouant un rôle essentiel» dans l'enseignement des langues fut finalement inscrite dans l'accord de Nouméa !!!

Le souci de développer en Océanie un enseignement visant à la formation d'enseignants locaux tant à Tahiti qu'en Nouvelle-Calédonie va déboucher à partir de 1980 sur la création des cursus repris aujourd'hui par l'université de Polynésie française et enfin admis - non sans de grandes résistances - à l'université et dans l'enseignement secondaire de NouvelleCalédonie.

C'est ainsi que Michel et Claude Lercari se succèdent alors à Nouméa et réussiront à enraciner l'idée de la nécessité d'enseigner cultures et civilisations locales ainsi que certaines des nombreuses langues. Pour réussir à implanter ces enseignements furent créés des « instituts», des «centres", appellations pompeuses pour de petites unités qui permettaient cependant de dispenser des cours. Michel fut responsable scientifique de l'Institut culturel mélanésien, puis directeur scientifique à l'Office culturel scientifique et technique canaque, enseigna au Centre de langues vernaculaires et à l'Institut des langues canaques afin d'assurer le DuLCO, (diplôme de
L.O. équivalent au DEUG) au travers de conventions avec le vice-rectorat de NouvelleCalédonie.

Aujourd'hui, Jacques Vernaudon, ancien élève de Michel, assure la relève et continue à lutter pour la qualité des enseignements de formation des «maitres » (quatre langues auront finalement été reconnues et admises au bac). Aux toutes premières thèses linguistiques préparées et soutenues en France par des océaniens, succèdent des travaux locaux. Michel, malheureusement, ne sera pas là pour les soutenances des thèses en cours. Son intérêt pour "ses " étudiants, son écoute, toujours disponible, son soutien énergique quand il le fallait (!) et l'effort constant pour pousser les candidats à suivre d'autres séminaires que les siens, à se « frotter la cervelle » à toutes les écoles possibles... tout cela, disparu, laisse les thésards " orphelins ».

En juin dernier, à Nouméa, Michel, passant près de l'endroit où fut l'un de ces centres où il avait fait cours, parlait avec une grande nostalgie de la « vieille » Lifou qui cultivait là son jardin. Elle le traitait comme l'un des jeunes lifous avec qui elle le voyait plaisanter et rire car Michel avait su entrer dans la classe d'âge des jeunes célibataires. Michel appréciait grandement leur côté hâbleur et irrévérencieux, les inventions folles et les aventures dans lesquelles ils s'entraînaient mutuellement. Eux aimaient ce «blanc» jovial, généreux et farceur qui pouvait, dans leur langue, dire des obscénités ou leur dévoiler les richesses de leur patrimoine oral. Ce sont eux d'ailleurs qui lui ont rendu hommage en plus grand nombre lors de la petite cérémonie d'adieux organisée à l'université de la NouvelleCalédonie.

$\mathrm{La}$ 《 vieille », donc, aimait à parler avec lui, elle lui racontait sa vie, lui transmettait tout un enseignement sur son île, lui confectionnait des spécialités locales et... des médicaments magiques!

Les Occidentaux et leur soi-disant cartésianisme ne passent pas indemnes par des cultures autres, mais Michel avait toujours su respecter les croyances et la foi, sous quelque forme de syncrétisme qu'elle se présente. Avec humour, il avait fait une place «à la magie kanak» qu'il pratiquait, à Paris, pour... trouver une place de parking! - et cela réussissait, bien sûr !

Bien que ce ne soit pas le lieu, ici, pour parler de ses recherches, le sujet méritant une étude complète évidemment, je dois cependant en rappeler les grands axes. Michel avait fait sienne la 
conviction de Piaget : notre corps est notre lieu de découverte du monde. Il posa alors l'hypothèse de correspondances entre les points d'articulation et le domaine du sens... À l'instar de Pierre Guiraud qui avait mis en lumière dans le français ces zones sonores où semble se nicher une « notion » (en empruntant à Antoine Culioli sa terminologie) comme /b/, par exemple, pour du « positif » (bon, beau, bien...) et /b-1/ pour des objets ronds (balle, bille, bulle, boule, bol...), Michel chercha dans les langues des étymons de signification. Ainsi, en tahitien /pa/ qui signifie surface, plat, paume et se retrouve dans toutes les actions faites avec la main ouverte, est un classificateur de poissons plats. Dans la plupart des langues austronésiennes, la voyelle /e/ (article, pronom, temps, aspect, " marqueur » des descriptions classiques) correspond à la simple présentation, à la mise en discours d'un élément, décrit un espace ou un temps, une réalité sur quoi rien n'est encore dit, alors que /a/ s'applique à une réalité que l'énonciateur prend en charge, connaît, définit, montre dans son discours. Cette approche que la relecture des textes océaniens semble justifier pleinement était une démarche audacieuse, car il est aussi périlleux de mettre en danger la linguistique « officielle » que de pénétrer sur un sol tabou!

Heureusement, le second domaine de sa recherche ne fut pas regardé par les chercheurs de l'oralité avec la même ironie dédaigneuse, au contraire, elle intéressa les spécialistes comme Jacques Dournes (qui travaillait sur le monde austronésien) ou Geneviève Calame-Griaule, membre de son jury de thèse, par exemple...

C'est peut-être la lecture de Rabelais qui initia Michel à la « double lecture ». Un texte contient d'autres textes possibles que celui qui se donne pour évident. Michel va étudier les répertoires de significations multiples des grands textes « classiques » tahitiens, des berceuses, des comptines, des textes fondateurs lifous, houailous... et en montrer les fonctions, pédagogiques, sociales autant que ludiques ou initiatiques. La création littéraire orale joue de toutes les formes sonores à sa disposition : mots, agencements, particules pour signifier dans un seul texte plusieurs messages. Clins d'œil complice aux initiés, un conte comme « le rat et le poulpe» étudié par Michel dans sa multiplicité de formes au travers de l'Austronésie, peut être un récit « historique » de l'installation d'un groupe humain, une allusion aux pratiques sexuelles, une cartographie des lieux, une mise en garde contre la pitié... aussi bien qu'un texte amusant.

Sa thèse d'État Les littératures océaniennes, approche syntaxique et stylistique. 'Le rat et le poulpe', communiquer, parler, raconter en Océanie est un monument de 1500 pages. Cette thèse soutenue en 2000 (Paris III, Sorbonne-Nouvelle, en Lettres et Sciences humaines, discipline Sciences du langage et ethnolinguistique) a obtenu les félicitations du jury à l'unanimité. Michel y a développé une partie de ses recherches et quand ce beau travail sera publié (en partie ou totalement), il fera référence.

Michel savait qu'il avait accompli une partie de ce qu'il avait à cœur pour les enseignements océaniens (en dépit des difficultés de tous ordre rencontrées), il voulait se dégager d'une partie de ses fonctions administratives et se consacrer à la rédaction de ses notes de terrain non encore publiées, développer les thèmes de sa recherche linguistique...

Mais voilà, tout est fini maintenant. Michel a été enterré au cimetière du Plessis-Robinson auprès de son père mort en début d'année, accompagné de nombreux amis et de sa mère sur un brancard d'hôpital. Ici, à Nouméa, une petite cérémonie en son honneur a eu lieu à l'université. Encore quelques articles et des hommages... et puis?

Il y a les amis qui se souviennent, bien sûr, mais aussi un très beau travail accompli qui, lui, survivra : une vraie recherche, originale, fondée sur le maniement des langues, permettant une meilleure compréhension du rôle du texte oral et une relecture de la littérature océanienne déjà collectée. Et, même si son nom est oublié peu à peu, son immense mérite est d'avoir contribué à la reconnaissance des cultures et des langues de Nouvelle-Calédonie, à l'enseignement de cellesci et par là même à la dignité d'humains qu'il appréciait.

\section{BIBLIOGRAPHIE}

1981. Phonologie et description grammaticale de l'anecom (Archipel du Vanuatu), thèse de doctorat de $3^{\mathrm{e}}$ cycle, non publiée.

1982. Langue et identité culturelle, Pacific 2000 1, Nouméa, Association pour le $4^{\mathrm{e}}$ festival des arts du Pacifique de Nouvelle Calédonie, pp. 27-30.

1987. Lexique zoologique et botanique anecomfrançais, Paris, INALCO, multigraphié.

1993. Hono ura ou la ceinture de pourpre, émergence de l'épopée polynésienne, Cahiers de littérature orale 32, pp. 125-155.

1994 (avec J. de La Fontinelle). Le corps de la parole: une nouvelle approche linguistique, Mwâ Véé 6 (Nouméa), pp. 29-33.

1995 (avec J. de La Fontinelle, J. Lynch \& P. Tepahae). La comparaison dans les langues d'Océanie: rup- 
ture, rapprochement, éloignement, Faits de langues 5, pp. 51-61.

1998 (avec D. Rey-Hulman). Du sens au sentiment, in Diana Rey-Hulman et Michel Boccara, Odeurs $d u$ monde. Écriture de la nuit, Paris, L'HarmattanINALCO.

2000. Les littératures océaniennes : approche syntaxique et stylistique, thèse de doctorat d'État Paris III - Sorbonne nouvelle, 1150 p. (disponible à l'ANRT : réf. 00PA030143 - http://www.anrtheses. com.fr/)

2001. Le petit colibri : analyse d'un texte oral mélanésien, Cahiers de littérature orale 50, pp. 34-46.

2002. Notes sur les messages végétaux en Océanie, JSO 114-115, pp. 223-227.
2002. La codification des langues de NouvelleCalédonie: des écritures missionnaires aux graphies linguistiques, in Caubet Dominique, Salem Chaker et Jan Sibille (éds), Codification des langues de France, actes du colloque Les langues de France et leur codifications (Paris, INALCO, 29-31 mai 2000), Paris, L'Harmattan, pp. 157-164.

2004. Comme le temps passe, Paris, Association internationale des belles-lettres, $40 \mathrm{p}$.

2004. Les langues océaniennes, Dossier Histoire et géographie 386 : Géohistoire Océanie, pp. 220-228.

2004. Les traditions orales, Dossier Histoire et géographie 386 : Géohistoire Océanie, pp. 229-242.

Michel Aufray et Michel Perret (éds), 1995. Cuisines d'Orient et d'ailleurs, Grenoble, éd. Glénat, 343 p. 